\title{
TPL-KATS-card sort: A tool for assessing structural knowledge
}

\author{
MICHELLE E. HARPER, FLORIAN G. JENTSCH, DEVON BERRY, \\ H. CATHY LAU, CLINT BOWERS, and EDUARDO SALAS \\ University of Central Florida, Orlando, Florida
}

\begin{abstract}
The study of how individuals organize knowledge has been a popular endeavor for several decades. As a result, techniques have been developed to assess how individuals represent and organize knowledge internally. Although several conceptual knowledge elicitation methods have been developed and used to assess the organization of knowledge, their use is often labor intensive and time consuming. Presented here is a software tool that was developed to reduce the problems associated with manually administering the conceptual knowledge elicitation technique, or card sorting. The TPL-KATS-card sort software not only simplifies the administration of the task, but also adds features to the card-sorting task such as media insertion, time stamping, and instructorless administration. In the present article, an introduction to the card-sorting technique is provided, the new software tool is described, and the advantages of the software are detailed.
\end{abstract}

Over the past several decades, applied cognitive psychologists have taken special interest in how individuals organize knowledge (see the Gentner \& Stevens study as cited in Jonassen, Beissner, \& Yacci, 1993; Quillian, 1968; Rumelhart, 1978; Rumelhart \& Ortony, 1982). From this interest arose the construct of structural knowledge, which refers to an internal representation and organization of information utilized by an individual. As a result of the interest in structural knowledge, assessment tools were developed to analyze people's internal representations. These assessment tools, known as structural knowledge elicitation techniques, not only represent an individual's knowledge but also check for potential inadequacies in knowledge and allow for assessment of knowledge shared within a team. Information of this type is of great importance because it can be used to identify problem areas so that they may be corrected. As a result of the application of these techniques, individuals can be trained to operate productively and without error.

Some frequently used methods of structural knowledge elicitation include similarity ratings, concept mapping, and card sorting (Beissner, 1992; Diekhoff \& Diekhoff, 1982; Geeslin \& Shavelson, 1975; Miller, 1969; Preece, 1976). These techniques are referred to as

This work was funded by Grant 99-G-047 from the Federal Aviation Administration (FAA), Office of the Chief Scientific and Technical Advisor for Human Factors, AAR-100, to the University of Central Florida, Partnership for Aviation Team Training Research. The opinions expressed in this article are those of the authors and do not necessarily represent the position of the University of Central Florida or the FAA. Correspondence concerning this article should be addressed to F. Jentsch, Team Performance Laboratory, Department of Psychology, University of Central Florida, P.O. Box 161390 Orlando, FL 328161390 (e-mail: fjentsch@ pegasus.cc.ucf.edu). conceptualmethods (Cooke, 1999) and rely on the use of domain concepts that are elicited either from the administrator or from the user. Examples of concepts from the domain of electricity, for instance, may include current, voltage, and circuit. Although the techniques all require users to manipulate concepts, they vary in the methods they use to assess structural knowledge. Similarity rating techniques use a method that requires the user to make a relatedness judgment between two pairs of concepts. Concept-mapping techniques require users to develop a map on the basis of how the concepts are related. Finally, card-sorting techniques rely on the user's placing the concepts into categories on the basis of their relatedness.

Whereas all of these techniques may elicit structural knowledge, one theory is that each technique may measure different aspects of structural knowledge. For example, Evans, Hitt, and Jentsch (2001) conducted a study on the convergence and sharedness of three techniques: card sorting, pairwise relatedness rating, and concept mapping. They found that when different individuals completed the same technique with the same concepts, the correlation between their outcomes was high. On the other hand, when the same individual completed all three techniques, the correlations between the outcomes were low.

Chi, Hutchinson, and Robin (1989) conducted a study that also supported the idea that different techniques could elicit different aspects of structural knowledge. Their study involved the creation of a map of an individual's knowledge about dinosaurs by having the participant generate a sequence of dinosaur names, identify dinosaurs with provided attributes, and generate attributes when given dinosaur names (see Chi \& Koeske, 1983, for more information). In addition to using the mapped network as a representation of knowledge, the participant also completed a card sort using dinosaur pictures. The 
outcomes of the two tasks revealed that the card-sorting task "was not predictable from the network representation" (Chi et al., 1989, p. 36), in other words, the outcome of the card sort and the outcome of the network map may represent different structures. Chi et al. suggested that through card sorting a higher level hierarchy, rather than the simple relationships represented by the network, may have been created. Further investigation of the networks revealed the same hierarchy as the card sort, but it is possible that without the card-sorting task, the higher order classification may have gone unnoticed.

As a result of the findings presented above, instead of viewing one technique as better than another at eliciting knowledge, one could look at each technique as part of a tool set. Using several tools to elicit knowledge may provide more information about an individual's structural knowledge than the use of only one tool.

However, for the purposes of this article our focus is on card sorting. Card sorting has been used in many fields. For example, Quilici and Mayer (2002) used card sorting to assess participants' knowledge of statistical word problems after exposure to different training methods. Niessen, Eyferth, and Bierwagen (1999) used card sorting to identify what cognitive structures allow experienced air traffic controllers to assess traffic situations. Also, card sorting has been used in the field of industrial education to assess the effectiveness of functional flow diagrams when used to train individuals on complex systems (Satchwell, 1997). Maiden and Hare (1998) used card sorting to aid in requirements engineering via the identification of conceptual categories associated with problem domains. Industrial psychologists have used card sorts to assess training needs (Goldstein, 1993). Furthermore, a form of card sort called Q-sort, which was introduced by Stephenson (1953), has been used to assess personality, attitude, and preference.

As was noted above, card sorting has been used in many domains and for many purposes. In the context of the present article, card sorting will be addressed as a tool that represents an individual's knowledge of a specific domain. Some researchers have questioned the validity of card sorting and its usefulness as a tool for eliciting structural knowledge. Other researchers believe the contrary. For example, as Fiore, Cuevas, and Oser (2003) note, "although a somewhat limited method, because users are forced to group together items rather rigidly ..., card sort data may be used to ascertain the degree to which one accurately views conceptual relations" (pp. 190-191; see also Cuevas, Fiore, \& Oser, 2002; Jonassen et al., 1993). Furthermore, Cheatham and Lane (2002) assessed the usefulness of knowledge elicitation techniques, including card sorting, and found that the card-sorting technique was efficient at capturing information and was found to be a better predictor of performance than the other tasks that were measured in the study.

\section{Psychometric Properties of Card Sort}

The psychometric validation of card sorting has been established by several researchers. Fiore, Fowlkes, Martin-
Milham, and Oser (2000) assessed the construct validity of card sorting by comparing it with similarity ratings using Pathfinder methodology (Schvaneveldt, 1990). The researchers found that these techniques are comparable when used to assess expert aviators' knowledge structures. In addition, Tessmer, Perrin, and Bennett (1998) studied card sort's reliability, that is, whether card sort would produce the same results each time it is administered to an individual. They concluded that "card sort can produce stable, coherent, measures of structural learning, if properly applied" (p. 74). More recently, Evans, Hitt, and Jentsch (2001) obtained similar results of moderate to high test-retest reliability $(r>.50)$, implying that an individual's structure is similar each time the assessment is repeated. Although some have pointed out that reliability coefficients of .50 and above are not sufficient, one must consider that low reliability may be a function not just of low measurement reliability, but also of the low reliability of the underlying construct. That is, it may be that novices in an area may just not have reliable mental models and that this is being revealed by repeated card sort measurements. Although reliability of the construct versus reliability of the measure is an important issue that should be addressed in future research, for the purpose of this article we believe that the reliability established by past research is sufficient.

Although card sorting has proven to be an effective tool for knowledge elicitation, few have addressed issues surrounding the administration and coding of the resultant sort. Thus, despite support for the technique, little effort has been made to create a more user-friendly way of executing the task. In the present article, in addition to describing the card-sorting technique, we respond to the need for a tool that is easier to use by discussing the development of software that computerizes card sorting, reduces the workload associated with the procedure, and optimizes the use of the tool.

\section{Executing Card Sorts}

Card-sorting techniques have been used as early as 1930, when A. R. Luria and the Russian psycholinguist Lev Vygotsky used them to investigate word meaning. During Luria and Vygotsky's study, participants viewed pictures of different items and were asked to sort them on the basis of their similarities. More modern uses of card sorting are quite similar in that they are intended to delineate an individual's structural knowledge through categorization of concepts in a given domain.

Procedures for completing the card-sorting task involve the placement of cards with concepts on them into piles on the basis of how the participant feels they are related. One common form of administration, known as a free sort, requires that the administrator provide the user with a set of index cards with concepts printed on them. The user then sorts the cards into as many piles that he or she deems necessary. After the user sorts the cards, the administrator may then ask the user to label the piles on the basis of their characteristics or content. Although this is a common form of administration, other variations 
exist. For example, administrators may place restrictions on the card sort by providing rules to the user on how to execute the card sort, such as by restricting the number of piles the user can make. In addition, administrators may restrain users by requiring them to place the cards into specified piles instead of allowing them to create their own piles on the basis of concepts. These are only a couple of examples of rules and restrictions; many other variations exist and are typically determined by the administrator's goals and interests (see Rao \& Katz, 1971).

\section{Scoring Card Sorts}

Just as the administration of card sorting varies, analysis of card-sorting outcomes also relies on different methods. The simplest and most frequently encountered of these variations entails a hit-or-miss scenario. If two concepts appear in the same pile, the pair receives a rating of 1; a pair of concepts that do not appear in the same pile receives a 0 . This type of scoring serves many purposes. For example, the hit-or-miss scenario can be used to compare users' card sorts with one another so that the variation or sharedness between their mental models may be assessed. In addition, and as discussed in further detail below, the hit-or-miss scoring method can be used to compare individuals' card sorts with referent or expert models. Other card sort scoring methods that have been implemented require the use of statistical techniques such as multidimensional scaling, hierarchical cluster analysis, or Pathfinder algorithms (Schvaneveldt, 1990).

\section{Evaluating Card Sorts}

Evaluation entails either assessing the characteristics of the piles that were made or reviewing the labels that were created for each pile. These assessments can reveal whether the participant sorted on the basis of structural features or hierarchical features. As was mentioned before, other card sort evaluations involve comparing the user's knowledge structure, or card sort model, with a referent or expert model. Referent or expert models represent the "ideal" card sort model for a given domain. Data has consistently shown that similarity to an expert model is positively related to performance on measures of knowledge acquisition (Fiore et al., 2003; Fiore, Cuevas, Scielzo, \& Salas, 2002). In addition to a performance measure, comparing a user's model to a referent or expert model can serve other evaluation purposes. For instance, the comparison can help administrators determine where the user is lacking knowledge or has inconsistent knowledge. Also, using a referent model allows for the evaluation of the differences between expert and novice card sorts. Smith-Jentsch, Campbell, Milanovich, and Reynolds (2001) conducted a study that required naval service personnel to complete a card sort on a given domain. The authors then compared the sorts with a referent model to determine how accurate the sailors' card sorts were as a function of experience and rank. The results of the study indicated that higher ranking indi- viduals had card sorts that more closely resembled the referent model than those of lower ranking individuals.

\section{Practical Concerns}

Despite the usefulness of card sorting as an experimental tool and training assessment aid, heretofore, its employment has been labor intensive and entailed numerous practical drawbacks. First, when card sorting was used in past experiments, a large number of participants would complete the card-sorting task, and in some cases they would complete it multiple times. As a result, the cardsorting task would require a substantial amount of time and human interaction. Participants had to be given cards in a predetermined or random order, and multiple, simultaneous administrations required multiple card sets. Second, the postadministration effort was even more labor intensive. After the administration and completion of the card sort by the user, the administrator was required to record the piles manually for future data entry, which also had to be done manually. These drawbacks of executing a card sort often created a time-consuming and laborious task and made it more susceptible to human error.

\section{TPL-KATS-CARD SORT SOFTWARE}

To the best of our knowledge, hitherto there has not been an automated form of card sort that makes the task user friendly by administering the task, recording the outcome, and analyzing the results. In response to this, The Team Performance Laboratory-Knowledge Analysis Testing Suite (TPL-KATS-card sort) software was developed in the Team Performance Laboratory at the University of Central Florida. In the remainder of this article, the new TPL-KATS-card sort software is described, recent studies in which it has been utilized are discussed, and the advantages of the software are detailed.

\section{System Requirements}

TPL-KATS-card sort is written in the Java programming language. The TPL-KATS-card sort software runs on any computer with a Java 1.2 virtual machine (see http://java.sun.com). Some platforms that incorporate Java 1.2 are Windows 95, 98, 2000, ME, and NT 4.0; Macintosh; Solaris; and Linux (see the Appendix for the requirements of different operating systems).

\section{Software Modes}

TPL-KATS-card sort has three modes of operation: User Mode, Administrator Mode, and Terminal Mode, respectively. In this context, the user is defined as the respondent and the administrator refers to the person who is administering the card-sorting task. The three modes are described below.

User Mode. Respondents perform the card-sorting task in this mode. The user interface comprises three major components: the board, the card list window, and the pile list window (see Figure 1). 


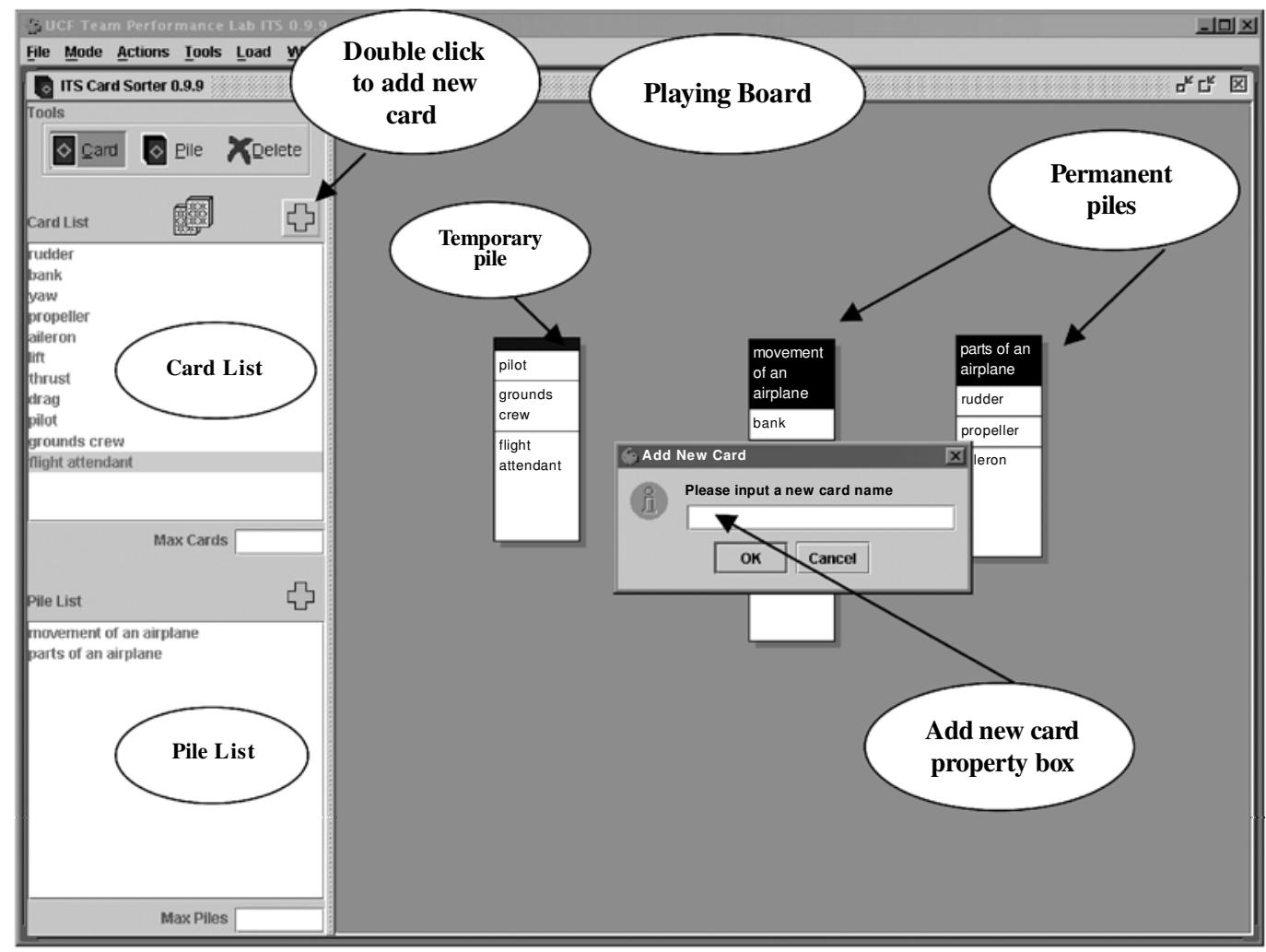

Figure 1. Components of interface with user and administrator options.

The board serves as the working space for the respondent. It is here that the respondent sorts and labels the cards. In order for the respondent to sort the cards, he or she needs to click on a particular card in the card list window, drag it over and then drop it onto the board in a manner similar to that used in the popular computer game Solitaire. As the respondent drags and drops the cards onto the board, the cards will be put into a temporary pile, which is indicated by a blue tab (see Figure 1). If the administrator prefers that the piles be labeled after the cards have been sorted, several methods may be used. The simplest way to label the piles is to double click on the blue tab located at the top of the pile of cards. Doing so will prompt a box in which the respondent can label the pile. Once labeled, the pile is considered permanent and the tab will then be black rather than blue. Although the pile is permanent, cards can still be moved into and out of the pile freely.

Administrator Mode. Administrator Mode is intended for the preparation of card-sorting tasks and is password protected. While in Administrator Mode, the administrator can set up the card-sorting task and the parameters related to the study. To begin, the administrator should create the concepts or cards to be sorted by the respondent. Clicking on the green plus sign above the card list will prompt an "Add New Card" property box in which each concept can be entered (see Figure 1). The administrator can generate as many cards as the system resources will allow.

Next, if the administrator prefers, he or she can specify pile labels so that the respondent can sort the cards only into the piles that are available. Adding specified piles is the same as creating concepts, except that the green plus sign above the pile list must be clicked on rather than that above the card list. For those administrators who prefer to use specified pile labels, TPL-KATS-card sort has a feature that allows the administrator to set a maximum pile limit. This feature ensures that the respondent is using the specified piles and is not creating additional ones.

In addition to being able to set limits on the number of piles, the administrator can also restrict the use of other features that are normally available. For example, each card and each pile has a property box that can be accessed by double clicking on the pile or card. Within the property box, the pile or card name can be changed, comments can be added, and the color of the text on the card can be changed (see Figure 2). If the administrator prefers to restrict the respondent from using these features, he or she may do so by clicking on and checking the box beside each feature. The benefit of the ability to restrict the use of features is that it keeps the respondents from changing the card and pile names, or making or deleting comments from the comment box. This feature is useful because it assures that the respondent is not al- 


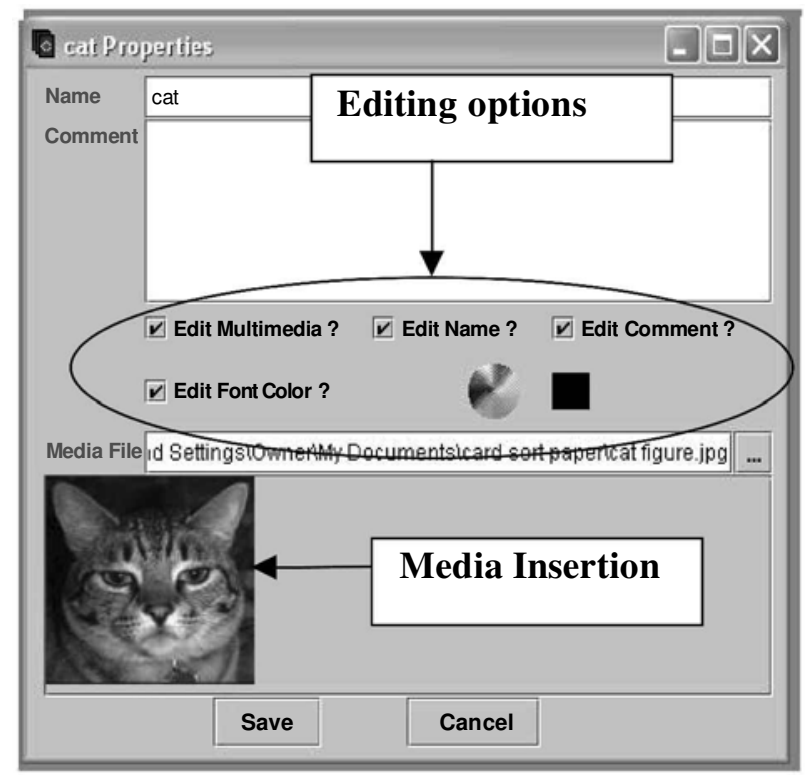

Figure 2. Property box displaying editing options and media insertion.

tering the parameters of the sort and that the sort is completed just as the administrator requests.

Terminal Mode. Terminal Mode is to be used by the respondent and is password protected. Only those respondents who are added as users by the administrator in Administrator Mode can access the Terminal Mode. While in Terminal Mode, the respondent has limited functions from which to choose. Those that are available allow the user to view a tutorial, start the card sort, and finish the card sort.

After a respondent has gained entry into Terminal Mode by using his or her user name and password, the software will prompt instructions on how to start and finish a card sort game. In addition, the software also prompts the respondent to indicate whether he or she would like instructions on how to complete the task (see discussion on the Help file, below). After the instructions are given, the respondent can begin the card sort game by accessing the drop-down File menu and clicking on Start. After the respondent has completed the game, he or she will click on Finish from the drop-down File menu. A prompt will provide the user with a debriefing of the card-sorting task. After the respondent has finished the game, the administrator can access the respondent's sort from Administrator Mode.

Terminal Mode is helpful to those who wish to use card sort for Internet or computer-based training sessions, classroom settings, and/or data collection. What makes the tool useful in these situations, for one thing, is that Terminal Mode is password protected: Only those respondents whom the administrator authorizes can access the card sort, and the sort can be completed by the respondent only one time. Also, the process of performing the card sort is simple for the respondent. Instructions on how to start the card sort and how to complete the task are provided, and following the task a debriefing form is displayed. A final useful feature in Terminal Mode is that, after the respondent is done with the sort, the software ensures that it was completed properly. If the card sort was not completed correctly, the respondent will not be allowed to save the sort. By not allowing respondents to save their sorts until they are complete, the software reduces the need to throw out incomplete data or to have respondents do the task more than once due to incompleteness.

Help file. TPL-KATS-card sort includes as a feature a text Help file that provides assistance to both the administrator and the respondent. The Administrator Mode contains instructions that include an introduction to the Administrator Mode screen. Also included are the relevant features used by the administrator, such as how to change the password, how to create cards and piles, and how to score the outcomes. In addition to the text Help file, there is a video Help file that includes instructions for the administrator and the respondent. Specific instructions, such as directions on how to create a card sort, how to execute a card sort, and how to insert media into the card sort, are provided within the video.

Instructions for using the software in User and Terminal Modes are provided for the respondent. User and Terminal Mode instructions consist of an introduction to the User or Terminal Mode screen as well as instructions on how to complete the card-sorting task. Other features that are pertinent in User and Terminal Modes, such as how to create pile names, how to arrange the cards and piles, and how to sort the cards, are also available in the Help file.

Logging actions. An added benefit of using this software to administer a card-sorting task is that the administrator has the capability of logging respondent actions with a time stamp. This feature provides further information to the administrator as to the exact sequence in which the participant sorted the concepts. Using this information in later analysis could help provide insight into areas in which a respondent's knowledge is weak or strong. For example, if a respondent makes a pile, labels it right away, and does not change it, as long as it is correctly sorted, one could assume that the respondent's knowledge about the corresponding concepts is stronger than his or her knowledge about those concepts that the respondent is hesitant in sorting.

Multimedia cards. A feature of the TPL-KATS-card sort software that makes it more powerful than the previous paper-and-pencil card-sorting procedure is the administrator's ability to insert multimedia files. Multimedia files include files of movies (.avi), pictures (.gif, .jpg), and sounds (.wav). These can be easily downloaded into the property box of a card and viewed by the respondent (see Figure 2 for an example of a property box with a .jpg insertion).

By using multimedia files, the administrator can create more elaborate studies and has the freedom to expand from the traditional methods of the card-sorting task. This feature may be useful, for example, in situations in 
which the experimenter is investigating how individuals categorize different sounds or animated sequences.

Data output and analysis. Five files are produced by the TPL-KATS-card sort software. The initial card sort file created by the experimenter is saved as a TPL-KATScard sort experiment file and can only be altered by the administrator. The card sort completed by the respondent is saved as a card sort file and allows the administrator to review a graphical depiction of exactly what the respondent's board looked like when the card sort procedure was completed.

To score the respondent's data once the task is completed, the administrator simply selects the Score function. Once this function is selected, the software creates three tabbed scoring files (see Figure 3). The first tabbed file is called the Card Legend and contains the codes that the software assigns to each concept (e.g., in Figure 3, apple was assigned the code $\mathrm{C} 0$ ). The second tabbed file is called the Pile Legend and indicates in which pile a particular card is located. The third tabbed file is called Scores and is a list of all possible pairs of cards or concepts. This file provides adjacency information by indicating whether the two components of each pair are grouped in the same pile. If they are in the same pile, the pair will be assigned a 1, and if not, the pair will be assigned a 0 . The Scores data file is available for direct analysis in statistical programs, such as Microsoft Excel.

Screen shots. Also included with the software is the capability to save screen shots. Screen shots are pictures of the playing board at any given time and can be used by prompting the EXPORT AS JPEG command in the file menu. To put it simply, this feature can save a picture of the playing board at any time during the user's card sort. These pictures can then be printed out and used as an additional aid for analysis.

\section{ADVANTAGES OF THE SOFTWARE}

TPL-KATS-card sort eliminates some of the more practical disadvantages of manual card sort while adding more flexibility to the task. Because of this, TPL-KATS-card sort software has an advantage over previously used manual card sort methods. In addition, Harper, Jentsch, Rhodenizer-VanDuyne, and Sanchez (2002) investigated the differences between administering card sorts manually and with TPL-KATS-card sort. After extensive comparisons, the researchers concluded that with respect to important psychometric measures, no differences exist between the two methods.

\section{Advantages in Data Collection}

As was mentioned before, when traditional card sorts were being prepared, administering the task and preparing the outcome for analysis was very tedious and labor intensive. With TPL-KATS-card sort, recording outcomes and entering them manually into additional software are no longer necessary. Instead, preparation is completed by the software seconds after the user has finished the task. The effect of this is not only a reduction in the time it takes to prepare the outcome for analysis, but also the elimination of any error caused by human data entry mistakes.

Another feature that adds to the ease of the software's administration is the incorporation of the Help files described above. Since instructions in the Help file are presented within the software, no instructions by the administrator are necessary. Additionally, there is an extensive on-line Help file for the card-sorting software that helps explain the task to the user.

The TPL-KATS-card sort tutorial and the Help file are beneficial in that they cut down on interaction between the administrator and the user. This type of benefit is even more useful when card sorts need to be performed in group settings (i.e., classrooms or job training facilities), in a machine-delivered training regiment (i.e., computer-based training), or via distance-learning methods such the Internet. The TPL-KATS-card sort tutorial and Help file limit interaction between the respondent and the administrator, thereby eliminating the need for multiple administrators and creating a more reliable task.

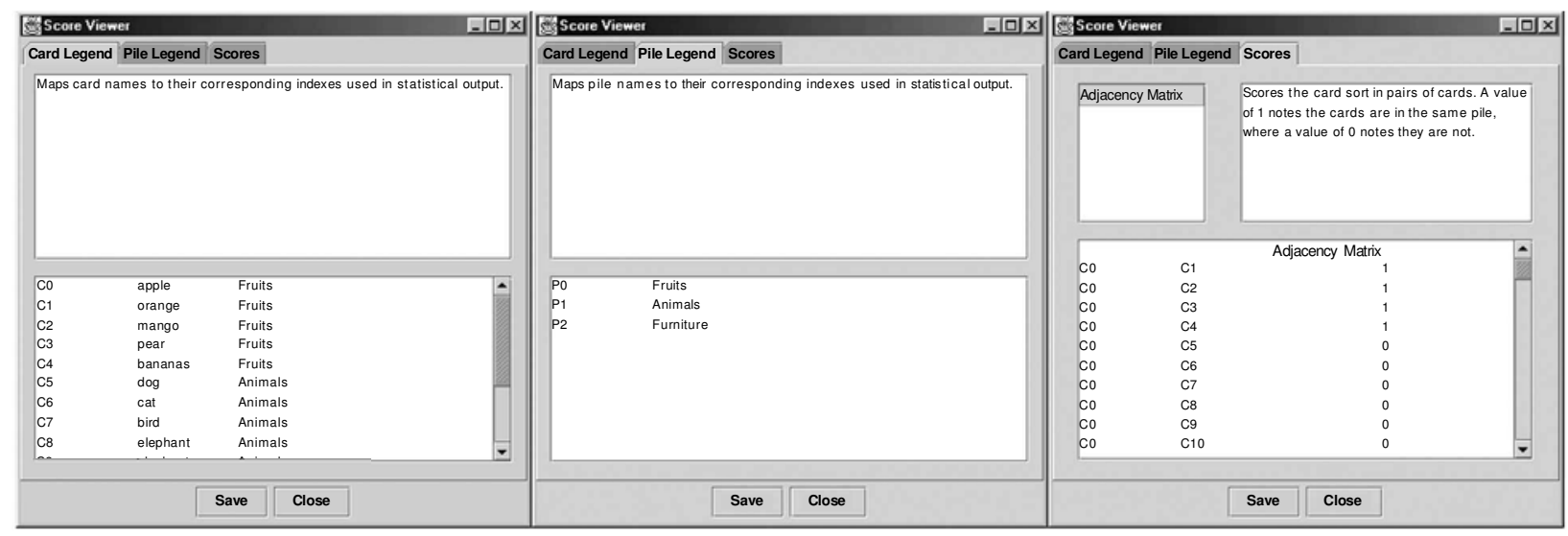

Figure 3. TPL-KATS-card sort Score files card. 
Finally, previous methods of administering card sorts relied on communication of the instructions by the administrator(s), thereby making the instructions more prone to inconsistencies due to human error and variation. TPL-KATS-card sort limits interaction between the administrator and the user, eliminating any error caused by lack of consistency in administrator instruction.

\section{Increased Flexibility}

Above and beyond the practical advantages stated in the preceding section, there are other benefits that make TPL-KATS-card sort a more flexible tool than the manual card-sorting procedures used heretofore. Two advantages discussed below are the ability of TPL-KATS-card sort to allow for media insertion and the accessibility of the software for instructorless administration via the World Wide Web.

Media insertion. Card sorts are typically limited to written "concept only" stimuli and are sometimes augmented by still pictures or graphics. TPL-KATS-card sort adds flexibility to the task by allowing other forms of stimuli (i.e., animation, motion pictures, and sounds) to be incorporated into the task.

The media insert function of TPL-KATS-card sort is useful for those researchers who, for several years, have been interested in how different stimuli are stored in memory (Job, Rumiati, \& Lotto, 1992). TPL-KATS-card sort, with its media insert capability, can make stimulus storage investigation easier by allowing different types of stimuli to be added to the card sort. Recent investigations of stimulus organization have used TPL-KATS-card sort (Evans, Hoeft, Jentsch, \& Bowers, 2001). In an attempt to determine how pictorial stimulus organization differs from textual stimulus organization, the researchers presented the participants with both textual and pictorial stimuli to sort. The researchers manipulated the way in which the participants sorted the different stimuli (free sort vs. structured sort). Evaluation entailed assessing the sharedness (i.e., how similar the sorts were) among the participants. The findings showed that in the free-sort textural stimulus condition, the participants' card sorts had the greatest sharedness. However, if the sort was structured, then the card sorts from the pictorial stimulus condition had the greatest sharedness. The investigators explain that the variance that is present within the freesort pictorial condition and absent within the structured pictorial condition may be the result of the numerous ways in which pictorial stimuli can be sorted when not constrained. As a result of these findings, the researchers concluded that using pictorial stimuli by themselves or with textural stimuli may add value to the meaning of a concept. In future research on the organization of stimuli, TPL-KATS-card sort could be used to investigate pictorial stimuli further. In addition, researchers can use the software to assess how stimuli such as sounds, music, or movie clips are stored in memory.

Machine-delivered assessment and distance administration. An additional advantage of using TPL-
KATS-card sort is that, as was explained above, the software's Terminal Mode allows the task to be used in a variety of settings, including machine-based (i.e., instructorless) and distance administration. Unlike previous manual versions of card sort, TPL-KATS-card sort requires no administrator in Terminal Mode. Considering the popularity of the Internet, Web-based classes, and Web-based data collection, TPL-KATS-card sort is a breakthrough for those who are looking for a training or classroom aid that is easily assessable and administered. In addition, TPL-KATS-card sort is perfect for those who are looking for a data collection tool that elicits knowledge from participants with little time and effort from the administrator.

\section{Summary and Availability}

The objective of the TPL-KATS-card sort software was to create a useful, inexpensive, user-friendly tool. These objectives were met by taking a well established structural knowledge elicitation technique and making it easy to use. Those interested in obtaining TPL-KATS-card sort may do so by first obtaining a user name and password from the authors and then visiting http://tpl.ucf.edu/. To begin downloading, click on Intelligent Training Suite found under the Software link on the home page.

\section{REFERENCES}

BEISSNER, K. L. (1992). Use of concept mapping to improve problem solving. Journal of Physical Therapy Education, 6, 22-27.

Cheatham, D. B., \& LAne, S. (2002). Differential access hypothesis: The effects of task and information type on the validity of knowledge acquisition methods. In Proceedings of the Forty-Sixth Meeting of the Human Factors \& Ergonomic Society (pp. 487-491). Baltimore, MD: Human Factors and Ergonomics Society.

Chi, M. T., Hutchinson, J. E., \& Robin, A. F. (1989). How inferences about novel domain-related concepts can be constrained by structural knowledge. Merrill-Palmer Quarterly, 35, 27-62.

CHI, M. T., \& KoESKE, R. D. (1983). Network representation of a child's dinosaur knowledge. Developmental Psychology, 19, 29-39.

Cooke, N. J. (1999). Knowledge elicitation. In F. T. Durso (Ed.), Applied cognition (pp. 479-509). Chichester, U.K.: Wiley.

Cuevas, H. M., Fiore, S. M., \& OSER, R. L. (2002). Scaffolding cognitive and metacognitive processes: Use of diagrams in computer-based training environments. Instructional Science, 30, 433-464.

Die KhofF, G. M., \& Die KHOFF, K. B. (1982). Cognitive maps as a tool in communicating structural knowledge. Educational Technology, 22, 28-30.

Evans, A. W., III, Hitt, J. M., II, \& Jentsch, F. (2001, March). Mental model reliability. Poster presented at the APA Division 21 and 19 Mid-year Meeting, Crystal City, VA.

Evans A. W., III, Hoeft, R., Jentsch, F., \& Bowers, C. (2001). Can a picture say a thousand words? Comparisons of structural organization vs. pictorial information. In Proceedings of the Forty-Sixth Annual Meeting of the Human Factors and Ergonomic Society (pp. 240244). Baltimore, MD: Human Factors and Ergonomics Society.

Fiore, S. M., Cuevas, H. M., \& OSER, R. L. (2003). A picture is worth a thousand connections: The facilitative effects of diagrams on task performance and mental model development. Computers in Human Behavior, 19, 185-199.

Fiore, S. M., Cuevas, H. M., Scielzo, S., \& SAlas, E. (2002). Training individuals for distributed teams: Problem solving assessment for distributed mission research. Computers in Human Behavior, 18, 125-140.

Fiore, S. M., Fowlkes, J., MARTin-Milham, L., \& OSER, R. L. (2000). 
Convergence or divergence of expert models: On the utility of knowledge structure assessment in training research. In Proceedings of the Forty-Fourth Annual Meeting of the Human Factors and Ergonomics Society (pp. 427-430). Santa Monica, CA: Human Factors and Ergonomics Society.

Geeslin, W. E., \& Shavelson, R. J. (1975). Comparison of content structure and cognitive structure in high school students' learning of probability. Journal of Research in Mathematics Education, 6, 109120.

Goldstein, I. L. (1993). Training in organization (3rd ed.). Pacific Grove, CA: Brooks/Cole.

Harper, M. E., Jentsch, F., Rhodenizer-Vanduyne, L. G., \& SANCHEZ, K. A. (2002). Computerized card sort training tool: Is it comparable to manual card sorting? In Proceedings of the Forty-Seventh Annual Meeting of the Human Factors and Ergonomic Society (pp. 2049-2053). Baltimore, MD: Human Factors and Ergonomics Society.

Job, R., Rumiati, R., \& Lotto, L. (1992). The picture superiority effect in categorization: Visual or semantic? Journal of Experimental Psychology: Learning, Memory, \& Cognition, 18, 1019-1028.

JonASSEN, D. H., BeISSNER, K., \& YACCI, M. (1993). Structural knowledge: Techniques for representing, conveying, and acquiring structural knowledge. Hillsdale, NJ: Erlbaum.

Maiden, N. A. M., \& HARE, M. (1998). Problem domain categories in requirements engineering. International Journal of Human-Computer Studies, 49, 281-304.

Miller, G. A. (1969). A psychological method to investigate verbal concepts. Journal of Mathematical Psychology, 6, 169-191.

Niessen, C., Eyferth, K., \& BierWAgen, T. (1999). Modeling cognitive processes of experienced air traffic controllers. Ergonomics, 42, 1507-1520.
PREECE,P. F. W. (1976). Mapping cognitive structure: A comparison of methods. Journal of Educational Psychology, 68, 1-8.

Quilici, J. H., \& MAYER, R. E. (2002). Teaching students to recognize structural similarities between statistics word problems. Applied Cognitive Psychology, 16, 325-342.

Quillian, M. R. (1968). Semantic memory. In M. Minsky (Ed.), Semantic information processing (pp. 216-260). Cambridge, MA: MIT Press.

RAO, V. R., \& KATZ, R. (1971). Alternative multidimensional scaling methods for large stimulus sets. Journal of Marketing Research, $\mathbf{8}$, 488-494.

RUMELHART, D. E. (1978). Schemata: The building blocks of cognition. In R. J. Spiro, B. C. Bruce, \& W. F. Brewer (Eds.), Theoretical issues in reading comprehension: Perspectives from cognitive psychology, linguistics, artificial intelligence, and education (pp. 45-95). Hillsdale, NJ: Erlbaum.

RUMELhART, D. E., \& ORTONY, A. (1982). The representation of knowledge in memory. Infancia y Aprendizaje, 19-20, 115-158.

SATCHWELL, R. E. (1997). Using functional flow diagrams to enhance technical systems understanding. Journal of Industrial Teacher Education, 34, 50-81.

SchVaneveldt, R. W. (1990). Pathfinder associative networks: Studies in knowledge organization. Norwood, NJ: Ablex.

Smith-Jentsch, K. A., Campbell, G. E., Milanovich, D. M., \& REYNOLDS, A. M. (2001). Measuring teamwork mental models to support training needs assessment, development, and evaluation: Two empirical studies. Journal of Organizational Behavior, 22, 179-194.

STE PHENSON, W. (1953). The study of behavior. Chicago: University of Chicago Press.

Tessmer, M., Perrin, B., \& Bennett, W. (1998). Assessing the stability of structural learning measures. Training Research Journal, 4, 57-77.

\section{APPENDIX \\ System Requirements}

Microsoft Windows 95, 98, 2000, ME, and NT 4.0. TPL-KATS-card sort is intended for use on Windows 95, Windows 98, Windows NT 4.0 with Service Pack 5, Windows ME, Windows 2000 Professional, Windows 2000 Server, and Windows 2000 Advanced Server operating systems running on Intel hardware. In non-English locales, only the Professional edition of Windows 2000 is supported. A Pentium 166-MHz or faster processor with at least $32 \mathrm{MB}$ of physical RAM are required. Forty-eight megabytes of RAM are recommended. Thirty megabytes of free disk space are needed for installation.

Macintosh operating system. TPL-KATS-card sort is intended for use in Mac OS 8.1 or higher operating environments. A 166-MHz or faster processor with at least $32 \mathrm{MB}$ of physical RAM is required. Fortyeight megabytes of RAM are recommended. Twenty-one megabytes of free disk space are needed for installation.

Solaris 2.6, 7, 8 operating environment. TPL-KATS-card sort is intended for use in Solaris 2.6, Solaris 7, and Solaris 8 operating environments. A Pentium 166-MHz or faster processor with at least $32 \mathrm{MB}$ of physical RAM is required. Forty-eight megabytes of RAM are recommended. Thirty-eight megabytes of free disk space are needed for installation.

Linux. TPL-KATS-card sort is supported on Intel Pentium platforms running the Linux kernel v2.2.12 and glibc v2.1.2-11 or later versions. A Pentium 166-MHz or faster processor with at least $32 \mathrm{MB}$ of physical RAM is required. Forty-eight MB of RAM, a 16-bit color mode, and KDE and KWM window managers used in conjunction with displays set to local hosts is recommended. Forty-two MB of free disk space is needed for installation. 